OPEN ACCESS

Edited by:

George Qian Li,

Western Sydney University, Australia

Reviewed by:

Xu-Dong Zhou,

Hunan University of Chinese Medicine,

China

Yan Lin,

Guizhou Medical University, China

*Correspondence:

Xia Yan

387808457@qq.com

Dawei Wang

davidwang33@139.com

Specialty section: This article was submitted to

Ethnopharmacology,

a section of the journal

Frontiers in Pharmacology

Received: 18 November 2020

Accepted: 24 February 2021

Published: 29 April 2021

Citation:

Wu K, Deng D, Yu B, Han Z, Huang L,

He $Y$, Yan $X$ and Wang $D$ (2021)

Evaluation of the Efficacy and Safety of

Chinese Herbal Injection Combined

With Trimetazidine for Viral Myocarditis:

A Network Meta-Analysis.

Front. Pharmacol. 12:630896.

doi: 10.3389/fphar.2021.630896

\section{Evaluation of the Efficacy and Safety of Chinese Herbal Injection Combined With Trimetazidine for Viral Myocarditis: A Network Meta-Analysis}

\author{
Kerui Wu ${ }^{1}$, Dingwei Deng ${ }^{2}$, Binghui $\mathrm{Yu}^{1}$, Ziyun Han ${ }^{1}$, Lanlin Huang ${ }^{1}$, Yaxing $\mathrm{He}^{1}$, Xia Yan ${ }^{2 *}$ \\ and Dawei Wang ${ }^{3 *}$

\begin{abstract}
${ }^{1}$ The Second Clinical College of Guangzhou University of Chinese Medicine, Guangzhou, China, ${ }^{2}$ The Second Affiliated Hospital of Guangzhou University of Chinese Medicine, Guangdong Provincial Hospital of Chinese Medicine, Guangzhou, China, ${ }^{3}$ Shunde Affiliated Hospital of Guangzhou University of Chinese Medicine, Shunde, China
\end{abstract}

Background: Viral myocarditis (VMC) is a common emergency of cardiovascular disease. Current treatment for VMC includes the prohibition of exercise plus supportive and symptomatic treatment, given the lack of specific antiviral therapeutic options and insufficient evidence for the use of novel immunosuppressive therapies. Trimetazidine, a drug used to improve myocardial energy metabolism, is frequently used for the treatment of viral myocarditis. In China, Chinese herbal injections ( $\mathrm{CH}$ s) are often used in combination with trimetazidine. Therefore, we evaluate the efficacy and safety of $\mathrm{CHI}$ combined with trimetazidine in the treatment of VMC through the method of network meta-analysis.

Methods: We searched PubMed, the Cochrane Library, Embase, China National Knowledge Infrastructure (CNKI), Wanfang Database, Chinese Scientific Journals Fulltext Database (VIP), and China Biology Medicine Database (CBM) databases from inception to September 1, 2020, to identify eligible randomized controlled trials. The Cochrane risk of bias tool was used to assess the risk of bias among selected studies and the Stata 16.0 software was used to perform the network meta-analysis.

Results: A total of 29 studies were included, representing data from 2,687 patients. The effectiveness rate, level of myocardial injury marker, and the adverse reaction rate were evaluated. Compared with conventional treatment or conventional treatment combined with trimetazidine, $\mathrm{CH}$ ls combined with trimetazidine appeared to have a better therapeutic effect, with higher effectiveness rate and better reduction of the levels of creatine kinase, creatine kinase-MB, and lactate dehydrogenase. Based on surface under the cumulative ranking, Shenmai injection combined with trimetazidine appeared to be superior in terms of effective rate, while Astragalus injection or Salviae miltiorrhizae and ligustrazine hydrochloride injection combined with trimetazidine appeared most effective in

\footnotetext{
Abbreviations: VMC, viral myocarditis; CHI, Chinese herbal injection; TCM, traditional Chinese medicine; RCTs, randomized controlled trials; CNKI, China National Knowledge Infrastructure; VIP, Chinese Scientific Journals Full-Text Database; CBM, China Biology Medicine; CK, creatine kinase; CK-MB, creatine kinase-MB; LDH, lactate dehydrogenase; cTnI, cardiac troponin I; OR, odds ratios; SMD, standard mean difference; CI, confidence intervals; IF, inconsistency factors; SUCRA, surface under the cumulative ranking.
} 
reducing myocardial injury markers. There was no significant difference in safety between the interventions. However, a lack of safety monitoring in some selected studies meant that the safety of some interventions could not be fully evaluated.

Conclusion: $\mathrm{CH}$ s combined with trimetazidine may have therapeutic value in the treatment of viral myocarditis, and Shenmai injection, Astragalus injection, and Salviae miltiorrhizae and ligustrazine hydrochloride injection may represent the most effective $\mathrm{CH}$ ls. Further clinical investigation is required to confirm these results.

Keywords: randomized controlled trials, network meta-analysis, viral myocarditis, trimetazidine, Chinese herbal injection

\section{INTRODUCTION}

Viral myocarditis (VMC) is a localized or diffuse myocardial inflammatory lesion caused by viral infection. Its clinical manifestations vary widely, from asymptomatic in mild cases to heart failure and even sudden cardiac death in severe cases. The most common manifestations are chest pain, heart failure, and fatigue dyspnea (Grün et al., 2012; Society of Cardiovascular Diseases, China Association of Chinese Medicine, 2020). Viral infection is believed to be the main cause of VMC, and the common viruses are coxsackievirus B, parvovirus B19, herpesvirus, and so on (Leone et al., 2019). Relevant epidemiological data show that the incidence of VMC is about $10-22$ per 100,000 people, and the population is mainly young and middle-aged (Global Burden of Disease Study 2013 Collaborators, 2015; Olejniczak et al., 2020). As severe cases of VMC can lead to heart failure and sudden cardiac death, which seriously affect the life of patients, the timely treatment of it is very important. Current treatment for viral myocarditis remains founded on the prohibition of exercise plus supportive and symptomatic treatment, given the lack of specific antiviral therapeutic options and insufficient evidence for the use of novel immunosuppressive therapies (Tschöpe et al., 2019).

Trimetazidine, an oxidation inhibitor of free fatty acids, is often used in the treatment of heart disease with the effect of improving myocardial energy metabolism and protecting myocardium (Marzilli et al., 2019). Some studies have shown that trimetazidine has a curative effect in the treatment of VMC, which can increase the clinical effectiveness rate, improve clinical symptoms, and promote the recovery of myocardial zymogram (Yu et al., 2014). In China, traditional Chinese medicine (TCM) also has significant advantages in the treatment of VMC, showing good therapeutic effects in anti-inflammation, protecting myocardium, enhancing immune function and so on (Cao et al., 2015). Chinese herbal injection (CHI), the product of the combination of traditional Chinese medicine and modern technology, is a kind of innovative preparation with high bioavailability and good curative effect (Li et al., 2017). It is an innovative application of Chinese medicine and has been widely used in the treatment of VMC. In recent years, a large number of clinical practices have combined trimetazidine with $\mathrm{CHI}$ in the treatment of VMC, showing a better therapeutic effect. Liu et al (2015) conducted a meta-analysis of the efficacy and safety of Astragalus injection combined with trimetazidine for VMC, and the results showed that compared with conventional treatment or conventional treatment combined with trimetazidine, Astragalus injection combined with trimetazidine improved the clinical efficacy and reduced the cardiac zymogram level significantly. Cheng (2015) researched the efficacy of trimetazidine combined with Shenmai injection in the treatment of VMC, and the results showed that the combination could significantly shorten the improvement time of clinical symptoms and signs, and reduced B-type natriuretic peptide (BNP) and C-reactive protein (CRP) levels. Wang (2019) showed that trimetazidine combined with Breviscapine injection in the treatment of acute VMC is superior to trimetazidine alone in controlling the level of inflammatory cytokines, improving myocardial zymogram and relieving symptoms and so on.

Although a variety of CHIs have shown considerable efficacy in the treatment of VMC, previous studies have only explored the efficacy and safety of a single $\mathrm{CHI}$ combined with trimetazidine. To date, no direct or indirect comparison of different CHIs combined with trimetazidine has been reported for the treatment of VMC, meaning that it remains unclear which CHIs are most effective in the treatment of this condition. In view of this, the study aims to indirectly compare the efficacy and safety of different CHIs combined with trimetazidine in the treatment of VMC though network meta-analysis and hopes to provide some reference for clinical treatment.

\section{MATERIALS AND METHODS}

\section{Inclusion Criteria}

All published clinical randomized controlled trials (RCTs) of CHI combined with trimetazidine in the treatment of VMC were selected. No restrictions were imposed on nationality, age, gender, and race. The control group was treated with conventional treatment or conventional treatment combined with trimetazidine. Conventional treatment included one or more of the following therapies: rest, sedation, antiarrhythmic therapy, myocardial protection, antioxidant therapy, antiviral therapy, and so on. The experimental group was treated with trimetazidine and $\mathrm{CHI}$ on the basis of the conventional treatment used in the control group. All the included literature should report any one of the primary or secondary outcome indicators. The primary outcome indicator was the effectiveness rate. The main reference criteria were as follows: markedly effective: 
clinical symptoms improved or disappeared, myocardial injury markers (myocardial zymogram or cardial troponin) returned to normal; effective: clinical symptoms relieved, myocardial injury markers improved partially, but did not fully return to normal; invalid: clinical symptoms did not improve or even further worsened, myocardial injury markers did not improve. Effectiveness rate $=\mathrm{N}$ (the number of markedly effective and effective cases) $/ \mathrm{N}$ (total number of cases) $\times 100 \%$. The secondary outcomes were as follows: 1) myocardial injury markers: creatine kinase (CK), creatine kinase-MB (CK-MB), lactate dehydrogenase $(\mathrm{LDH})$, and cardiac troponin I (cTnI); 2) adverse reactions.

\section{Exclusion Criteria}

1) Studies on treatment combined with other TCM-related treatment measures, such as TCM decoction, Chinese patent medicines, and acupuncture were excluded; 2) participants complicated with other diseases, such as coronary heart disease and diabetes; 3) literature works published with duplicate data; 4) the reported data were incomplete and impossible to be acquired; 5) the reported data were inconsistent with the conclusion.

\section{Search Strategies}

To obtain RCTs of CHI combined with trimetazidine in the treatment of VMC, we searched PubMed, the Cochrane Library, Embase, China National Knowledge Infrastructure (CNKI), Wanfang database, Chinese Scientific Journals FullText Database (VIP), and China Biology Medicine Database (CBM) from inception to September 1, 2020. The search terms in English database were as follows: "Viral myocarditis," "Myocarditides," "Carditis," "Myocarditis," "Trimetazidine," "Centrophène," "Trimetazidine Dihydrochloride," "Dihydrochloride, "Trimetazidine," "Vastarel," "Trimétazidine Irex," "Vasartel," and "Idaptan." The search terms in Chinese database included "bing du xing xin ji yan," "qu mei ta qin," "yan suan qu mei ta qin," "wan shuang li," and "san jia yang bian qin." The search strategy of each database is shown in Supplementary Table S1.

\section{Literature Screening and Data Extraction}

After literature retrieval, two evaluators independently conducted literature screening according to the inclusion and exclusion criteria. A preliminary screening was carried out according to the title and abstract, and then rescreening was carried out by reading the full text. Any differences in the screening results of the two evaluators were resolved by discussion with a third evaluator. After determining the included studies, the data of literature was extracted as follows: title, authors, year of publication, baseline status, methodological information, sample size, intervention measures, the course of treatment, and outcomes.

\section{Bias Risk Assessment}

Two evaluators independently assessed the risk of bias in the included studies by using the Cochrane risk of bias tool (Higgins et al., 2011), which consisted of the following items: 1) random sequence generation; 2) allocation hiding; 3) blinding of outcome evaluators; 4) blinding of patients and trial personnel; 5) incomplete result data; 6) selective reporting; and 7) other biases. The risk assessment criteria are divided into low, high, and uncertain bias risk. Any differences in the assessment results of the two evaluators were resolved by discussion with a third evaluator. The bias risk assessment results of the included studies were visualized by RevMan5.3 software.

\section{Statistical Analysis}

All statistical analysis was performed using Stata 16.0 software (Stata Company). For dichotomous variables (effectiveness rate and adverse reaction rate), odds ratios (OR), and 95\% confidence intervals (CI) were used to assess effect size, whereas the effect size of continuous variables (myocardial injury markers) was assessed using the standard mean difference (SMD) and 95\% CI. Considering the expected heterogeneity in the included studies, a random-effects model was used for statistical analysis. If the data could not be meta-analyzed, we would conduct a descriptive analysis. Based on the classical frequency, the random-effects model was selected under the "Network" command in the analysis software to perform the network meta-analysis. A network diagram of interventions was constructed to show the relationships between interventions. Where no closed loop was formed, the consistency model was used for analysis. When a closed loop was observed, an overall inconsistency check was performed. If $p$ value $>0.05$, it indicated that the overall inconsistency was not significant, otherwise, it indicated that the overall inconsistency was significant. At the same time, the inconsistency test of the loop was carried out to determine inconsistency between direct and indirect evidence by calculating the absolute difference between these types of evidence, expressed as an inconsistency factor (IF). Whereas 95\% CI of the IF values contained 0 , and the inconsistency of direct and indirect evidence was considered not significant. Otherwise, the inconsistency was deemed significant. When no inconsistency was apparent, the consistency model was used for analysis. Otherwise, the inconsistency model was selected for analysis. The value of surface under the cumulative ranking (SUCRA) was subsequently calculated and the number of iterations set at 5,000. Intervention measures were sorted based on SUCRA value. The larger the SUCRA value, the higher the ranking, indicating that the intervention was more likely to be the best intervention. Funnel plots were used to evaluate publication bias in the included studies.

\section{RESULTS}

\section{Literature Search and Screening}

A total of 904 literature works were obtained. Among them, there were 3 from PubMed, 7 from the Cochrane Library, 4 from Embase, 453 from CNKI, 172 from Wanfang database, 135 from VIP, and 130 from CBM. After screening, a total of 29 studies were included. The literature screening process is shown in Figure 1. 


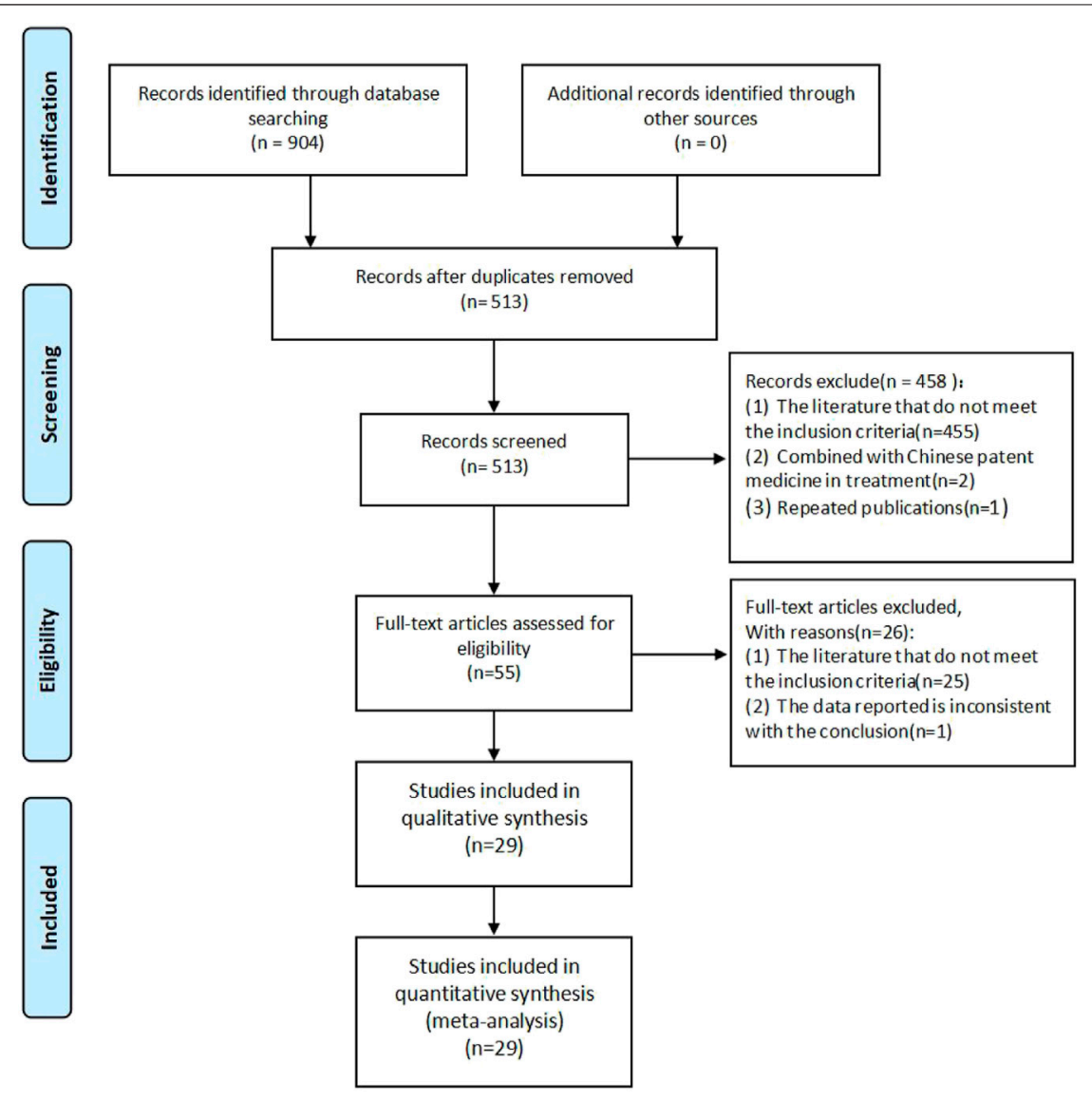

FIGURE 1 | Flow diagram of literature screening process.

\section{Basic Characteristics of Included Studies}

29 studies enrolled a total of 2,687 patients. All the included studies were conducted in China, and the literature was published from 2009 to 2020. The baselines of all studies were comparable between the experimental and control groups. In terms of intervention measures, the control groups of 18 studies were treated with conventional treatment alone, whereas the experimental groups were treated with $\mathrm{CHI}$ and trimetazidine on the basis of the control groups. Among them, 14 studies were combined with Astragalus injection, 3 studies combined with Shenfu injection, and 1 study combined with Shenmai injection. In the other 11 studies, the control groups were treated with conventional treatment combined with trimetazidine, whereas the experimental groups were treated with $\mathrm{CHI}$ on the basis of the control group. Among them, 4 studies combined with Astragalus injection, 4 studies combined with Salviae miltiorrhizae and ligustrazine hydrochloride injection, 2 studies combined with Breviscapine injection, and 1 study combined with Shenmai injection. The detailed information of CHIs used in included studies and the chemical analysis of them are shown in Supplementary Tables S2, S3. In the course of treatment, the shortest was 2 weeks and the longest was 8 weeks. In the outcome indicators, 25 studies reported the effectiveness rate, 10 studies reported the level of CK, 12 studies reported the level of CK-
$\mathrm{MB}, 13$ studies reported the level of $\mathrm{LDH}, 8$ studies reported the level of cTnI, and 20 studies reported the adverse reactions during treatment. The basic characteristics of included studies are detailed in Table 1. For the convenience of description, A refers to conventional treatment, $\mathrm{B}$ refers to conventional treatment combined with trimetazidine and Astragalus injection, $\mathrm{C}$ refers to conventional treatment combined with trimetazidine and Shenfu injection, D refers to conventional treatment combined with trimetazidine, E refers to conventional treatment combined with trimetazidine and Salviae miltiorrhizae and ligustrazine hydrochloride injection, $\mathrm{F}$ refers to conventional treatment combined with trimetazidine and Breviscapine injection, and $\mathrm{G}$ refers to conventional treatment combined with trimetazidine and Shenmai injection. The network diagrams of the seven interventions in different outcome indicators are shown in Figure 2.

\section{Bias Risk Assessment of Included Studies}

The methodological quality of the included studies was generally low. In the generation of random sequences, 9 studies used random number tables, and 1 study used lottery randomization, so they were at low bias risk. Other studies only mentioned randomness and did not describe random methods, so they were at uncertain bias risk. None studies 
TABLE 1 | Basic characteristics of the included studies.

\begin{tabular}{|c|c|c|c|c|c|c|}
\hline \multirow[t]{2}{*}{ Study } & \multirow{2}{*}{$\begin{array}{c}\text { Age (mean } \\
\text { or range) } \\
\text { C/E }\end{array}$} & \multirow{2}{*}{$\begin{array}{c}\text { Sample } \\
\text { size } \\
\text { C/E }\end{array}$} & \multicolumn{2}{|r|}{ Intervention } & \multirow[t]{2}{*}{ Courses } & \multirow[t]{2}{*}{ Outcome } \\
\hline & & & C & $\mathbf{E}$ & & \\
\hline Chen (2011) & $17-46 / 17-46$ & $32 / 32$ & Conventional treatment & $\begin{array}{l}\text { Conventional treatment }+ \text { trimetazidine } 20 \mathrm{mg} / \mathrm{d}+ \\
\text { Astragalus injection } 40 \mathrm{ml} / \mathrm{d}\end{array}$ & $\begin{array}{l}\text { Astragalus } \\
\text { injection } 2 \mathrm{w} \\
\text { Others } 8 \mathrm{w}\end{array}$ & (1) (4) (6) \\
\hline Chen (2014) & $57.8 / 57.8$ & $80 / 80$ & Conventional treatment & $\begin{array}{l}\text { Conventional treatment }+ \text { trimetazidine } 20 \mathrm{mg} / \mathrm{d}+ \\
\text { Astragalus injection } 50 \mathrm{ml} / \mathrm{d}\end{array}$ & $1 \mathrm{~m}$ & (1) (6) \\
\hline Dai (2018) & $\begin{array}{l}25.4 \pm 8.6 / \\
25.6 \pm 7.9\end{array}$ & $20 / 20$ & Conventional treatment & $\begin{array}{l}\text { Conventional treatment }+ \text { trimetazidine } 60 \mathrm{mg} / \mathrm{d}+ \\
\text { Astragalus injection } 50 \mathrm{ml} / \mathrm{d}\end{array}$ & $1 \mathrm{~m}$ & $\begin{array}{l}\text { (1) }(2)(3) \\
\text { (4) (6) }\end{array}$ \\
\hline Ge,et al. (2010) & $\begin{array}{l}27.2 \pm 11.2 / \\
26.8 \pm 10.7\end{array}$ & $30 / 30$ & Conventional treatment & $\begin{array}{l}\text { Conventional treatment }+ \text { trimetazidine } 20 \mathrm{mg} / \mathrm{d}+ \\
\text { Astragalus injection } 40 \mathrm{ml} / \mathrm{d}\end{array}$ & $\begin{array}{l}\text { Astragalus } \\
\text { injection } 2 \mathrm{w} \\
\text { Others } 8 \mathrm{w}\end{array}$ & (1) (6) \\
\hline Ma (2012) & $\begin{array}{l}22.48 \pm 7.2 / \\
23.56 \pm 8.5\end{array}$ & $46 / 62$ & Conventional treatment & $\begin{array}{l}\text { Conventional treatment }+ \text { trimetazidine } 60 \mathrm{mg} / \mathrm{d}+ \\
\text { Astragalus injection } 20 \mathrm{~g} / \mathrm{d}\end{array}$ & $2 w$ & (1) (3) (5) (6) \\
\hline $\mathrm{Pu}(2013)$ & - & $67 / 79$ & Conventional treatment & $\begin{array}{l}\text { Conventional treatment }+ \text { trimetazidine } 60 \mathrm{mg} / \mathrm{d}+ \\
\text { Astragalus injection } 50 \mathrm{ml} / \mathrm{d}\end{array}$ & $4 w$ & (1) (6) \\
\hline $\begin{array}{l}\text { Shao,et al. } \\
(2012)\end{array}$ & $27 \pm 11 / 28 \pm 7$ & $48 / 50$ & Conventional treatment & $\begin{array}{l}\text { Conventional treatment }+ \text { trimetazidine } 60 \mathrm{mg} / \mathrm{d}+ \\
\text { Astragalus injection } 10-20 \mathrm{ml} / \mathrm{d}\end{array}$ & $\begin{array}{l}\text { Astragalus } \\
\text { injection } 2 \mathrm{w} \\
\text { Others } 8 \mathrm{w}\end{array}$ & (1) (6) \\
\hline Sun (2013) & $30 \pm 5 / 28 \pm 5$ & $40 / 40$ & Conventional treatment & $\begin{array}{l}\text { Conventional treatment }+ \text { trimetazidine } 60 \mathrm{mg} / \mathrm{d}+ \\
\text { Astragalus injection } 20 \mathrm{~g} / \mathrm{d}\end{array}$ & $4 w$ & (1) (6) \\
\hline Wang (2016) & $\begin{array}{r}55.6 \pm 2.4 / \\
56.3 \pm 2.8\end{array}$ & $37 / 37$ & Conventional treatment & $\begin{array}{l}\text { Conventional treatment }+ \text { trimetazidine } 60 \mathrm{mg} / \mathrm{d}+ \\
\text { Astragalus injection } 20 \mathrm{ml} / \mathrm{d}\end{array}$ & $2 w$ & $(1)(3)(5)(6)$ \\
\hline Wang (2010) & $31 \pm 10 / 30 \pm 10$ & $50 / 50$ & Conventional treatment & $\begin{array}{l}\text { Conventional treatment }+ \text { trimetazidine } 60 \mathrm{mg} / \mathrm{d}+ \\
\text { Astragalus injection } 30 \mathrm{ml} / \mathrm{d}\end{array}$ & $3 w$ & (1) (6) \\
\hline $\begin{array}{l}\text { Xu and Zhang } \\
\text { (2011) }\end{array}$ & $14-40 / 13-41$ & $30 / 60$ & Conventional treatment & $\begin{array}{l}\text { Conventional treatment }+ \text { trimetazidine } 60 \mathrm{mg} / \mathrm{d}+ \\
\text { Astragalus injection } 40 \mathrm{ml} / \mathrm{d}\end{array}$ & $2 w$ & (1) (4) \\
\hline Yang (2009) & $31 \pm 10 / 32 \pm 10$ & $43 / 45$ & Conventional treatment & $\begin{array}{l}\text { Conventional treatment }+ \text { Trimetazidine } 60 \mathrm{mg} / \mathrm{d}+ \\
\text { Astragalus injection } 50 \mathrm{ml} / \mathrm{d}\end{array}$ & $4 w$ & (1) (6) \\
\hline $\begin{array}{l}\text { Zhang,et al. } \\
\text { (2015) }\end{array}$ & $\begin{array}{l}25.2 \pm 8.5 / \\
26.2 \pm 8.5\end{array}$ & $30 / 30$ & Conventional treatment & $\begin{array}{l}\text { Conventional treatment }+ \text { trimetazidine } 60 \mathrm{mg} / \mathrm{d}+ \\
\text { Astragalus injection } 30 \mathrm{ml} / \mathrm{d}\end{array}$ & - & (1) \\
\hline Yu (2014) & $\begin{array}{l}32.4 \pm 5.6 / \\
32.9 \pm 6.1\end{array}$ & $51 / 51$ & Conventional treatment & $\begin{array}{l}\text { Conventional treatment }+ \text { trimetazidine } 60 \mathrm{mg} / \mathrm{d}+ \\
\text { Astragalus injection } 50 \mathrm{ml} / \mathrm{d}\end{array}$ & $4 w$ & $(1)(3)(5)(6)$ \\
\hline $\begin{array}{l}\text { Zhang,et al. } \\
(2016)\end{array}$ & $\begin{array}{l}44.1 \pm 7.2 / \\
44.5 \pm 7.8\end{array}$ & $40 / 40$ & Conventional treatment & $\begin{array}{l}\text { Conventional treatment }+ \text { trimetazidine } 60 \mathrm{mg} / \mathrm{d}+ \\
\text { Shenfu injection } 50 \mathrm{ml} / \mathrm{d}\end{array}$ & $4 w$ & (1) (2) (3) (4) \\
\hline $\begin{array}{l}\text { Sun and Sun } \\
(2018)\end{array}$ & $\begin{array}{l}53.1 \pm 5.8 / \\
52.4 \pm 5.3\end{array}$ & $51 / 51$ & Conventional treatment & $\begin{array}{l}\text { Conventional treatment + trimetazidine } 60 \mathrm{mg} / \mathrm{d}+ \\
\text { Shenfu injection } 50 \mathrm{ml} / \mathrm{d}\end{array}$ & $\begin{array}{l}\text { Shenfu } \\
\text { injection } 2 \mathrm{w} \\
\text { Others } 4 \mathrm{w}\end{array}$ & (1) \\
\hline Gao (2019) & $\begin{array}{l}46.21 \pm 2.57 / \\
46.73 \pm 2.10\end{array}$ & $39 / 39$ & Conventional treatment & $\begin{array}{l}\text { Conventional treatment }+ \text { trimetazidine } 60 \mathrm{mg} / \mathrm{d}+ \\
\text { Shenfu injection } 40-200 \mathrm{ml} / \mathrm{d}\end{array}$ & - & $(1)(2)(3)(4)$ \\
\hline $\begin{array}{l}\text { Pang and Huang } \\
\text { (2013) }\end{array}$ & $\begin{array}{l}10 m-12 / \\
10 m-12\end{array}$ & $33 / 33$ & Conventional treatment & $\begin{array}{l}\text { Conventional treatment }+ \text { trimetazidine } \\
0.3-0.5 \mathrm{mg} / \mathrm{kg} / \mathrm{d}+\text { Shenmai injection } 0.5-1 \mathrm{ml} / \mathrm{kg} / \mathrm{d}\end{array}$ & $2 w$ & (1) \\
\hline Wang (2012) & $60 \pm 9 / 55 \pm 11$ & $35 / 35$ & $\begin{array}{l}\text { Conventional treatment }+ \\
\text { trimetazidine } 60 \mathrm{mg} / \mathrm{d}\end{array}$ & $\begin{array}{l}\text { Conventional treatment }+ \text { trimetazidine } 60 \mathrm{mg} / \mathrm{d}+ \\
\text { Astragalus injection } 50 \mathrm{ml} / \mathrm{d}\end{array}$ & $4 w$ & (1) (6) \\
\hline Zheng (2019) & $\begin{array}{l}49.15 \pm 16.18 / \\
48.47 \pm 15.25\end{array}$ & $44 / 45$ & $\begin{array}{l}\text { Conventional treatment }+ \\
\text { trimetazidine } 60 \mathrm{mg} / \mathrm{d}\end{array}$ & $\begin{array}{l}\text { Conventional treatment }+ \text { trimetazidine } 60 \mathrm{mg} / \mathrm{d}+ \\
\text { Astragalus injection } 10-20 \mathrm{ml} / \mathrm{d}\end{array}$ & $6 w$ & (3) (4) (6) \\
\hline Cui (2018) & $\begin{array}{l}55.2 \pm 6.5 / \\
54.8 \pm 6.3\end{array}$ & $35 / 35$ & $\begin{array}{l}\text { Conventional treatment }+ \\
\text { trimetazidine } 60 \mathrm{mg} / \mathrm{d}\end{array}$ & $\begin{array}{l}\text { Conventional treatment }+ \text { trimetazidine } 60 \mathrm{mg} / \mathrm{d}+ \\
\text { Astragalus injection } 40 \mathrm{ml} / \mathrm{d}\end{array}$ & $4 w$ & (2) (3) (4) (5) \\
\hline Wei,et al. (2020) & $\begin{array}{l}70.3 \pm 7.8 / \\
70.9 \pm 7.5\end{array}$ & $90 / 90$ & $\begin{array}{l}\text { Conventional treatment }+ \\
\text { trimetazidine } 60 \mathrm{mg} / \mathrm{d}\end{array}$ & $\begin{array}{l}\text { Conventional treatment }+ \text { trimetazidine } 60 \mathrm{mg} / \mathrm{d}+ \\
\text { Astragalus injection } 20 \mathrm{ml} / \mathrm{d}\end{array}$ & $2 w$ & $\begin{array}{l}\text { (1) (3) (4) } \\
\text { (5) (6) }\end{array}$ \\
\hline Zhu,et al. (2020) & $\begin{array}{c}5.81 \pm 1.22 / \\
5.7 \pm 1.15\end{array}$ & $46 / 46$ & $\begin{array}{l}\text { Conventional treatment }+ \\
\text { trimetazidine } 20 \mathrm{mg} / \mathrm{d}\end{array}$ & $\begin{array}{l}\text { Conventional treatment + trimetazidine } 20 \mathrm{mg} / \mathrm{d}+ \\
\text { Salviae miltiorrhizae and ligustrazine hydrochloride } \\
\text { injection } 5 \mathrm{ml} / \mathrm{d}\end{array}$ & $30 \mathrm{~d}$ & $\begin{array}{l}\text { (1) }(2)(3)(4) \\
\text { (5) (6) }\end{array}$ \\
\hline Wang (2020) & $\begin{array}{l}9.91 \pm 1.27 / \\
9.89 \pm 1.31\end{array}$ & $50 / 50$ & $\begin{array}{l}\text { Conventional treatment }+ \\
\text { trimetazidine } 60 \mathrm{mg} / \mathrm{d}\end{array}$ & $\begin{array}{l}\text { Conventional treatment + trimetazidine } 60 \mathrm{mg} / \mathrm{d}+ \\
\text { Salviae miltiorrhizae and ligustrazine hydrochloride } \\
\text { injection } 5 \mathrm{ml} / \mathrm{d}\end{array}$ & $30 \mathrm{~d}$ & (2) (3) (4) (5) \\
\hline $\begin{array}{l}\text { Chen and Zeng } \\
\text { (2018) }\end{array}$ & $\begin{array}{l}41.2 \pm 6.8 / \\
41.8 \pm 6.9\end{array}$ & $49 / 49$ & $\begin{array}{l}\text { Conventional treatment }+ \\
\text { trimetazidine } 60 \mathrm{mg} / \mathrm{d}\end{array}$ & $\begin{array}{l}\text { Conventional treatment + trimetazidine } 60 \mathrm{mg} / \mathrm{d}+ \\
\text { Salviae miltiorrhizae and ligustrazine hydrochloride } \\
\text { injection } 5 \mathrm{ml} / \mathrm{d}\end{array}$ & $1 \mathrm{~m}$ & (1) (2) (4) \\
\hline $\begin{array}{l}\text { Li and Gao } \\
\text { (2016) }\end{array}$ & $\begin{array}{l}43.9 \pm 7.9 / \\
43.3 \pm 7.5\end{array}$ & $55 / 55$ & $\begin{array}{l}\text { Conventional treatment + } \\
\text { trimetazidine } 20 \mathrm{mg} / \mathrm{d}\end{array}$ & $\begin{array}{l}\text { Conventional treatment + trimetazidine } 20 \mathrm{mg} / \mathrm{d}+ \\
\text { Salviae miltiorrhizae and ligustrazine hydrochloride } \\
\text { injection } 5 \mathrm{ml} / \mathrm{d}\end{array}$ & $30 \mathrm{~d}$ & $(1)(2)(4)(6)$ \\
\hline Miao (2019) & $51.17 \pm 2.67 /$ & $49 / 49$ & Conventional treatment + & Conventional treatment + trimetazidine $60 \mathrm{mg} / \mathrm{d}+$ & $1 \mathrm{~m}$ & (2) (6) \\
\hline
\end{tabular}


TABLE 1 | (Continued) Basic characteristics of the included studies.

\begin{tabular}{|c|c|c|c|c|c|c|}
\hline \multirow[t]{2}{*}{ Study } & \multirow{2}{*}{$\begin{array}{c}\text { Age (mean } \\
\text { or range) } \\
\text { C/E }\end{array}$} & \multirow{2}{*}{$\begin{array}{c}\begin{array}{c}\text { Sample } \\
\text { size }\end{array} \\
\text { C/E }\end{array}$} & \multicolumn{2}{|r|}{ Intervention } & \multirow[t]{2}{*}{ Courses } & \multirow[t]{2}{*}{ Outcome } \\
\hline & & & C & $\mathbf{E}$ & & \\
\hline Wang (2017) & $\begin{array}{l}42.1 \pm 15.9 / \\
43.2 \pm 15.6\end{array}$ & $62 / 62$ & $\begin{array}{l}\text { Conventional treatment }+ \\
\text { trimetazidine } 60 \mathrm{mg} / \mathrm{d}\end{array}$ & $\begin{array}{l}\text { Conventional treatment }+ \text { trimetazidine } 60 \mathrm{mg} / \mathrm{d}+ \\
\text { Breviscapine injection } 5 \mathrm{ml} / \mathrm{d}\end{array}$ & $4 w$ & $\begin{array}{c}(1)(2)(3)(4) \\
\text { (5) (6) }\end{array}$ \\
\hline $\mathrm{He}(2014)$ & $\begin{array}{l}33.52 \pm 1.14 / \\
33.52 \pm 1.14\end{array}$ & $30 / 30$ & $\begin{array}{l}\text { Conventional treatment }+ \\
\text { trimetazidine } 60 \mathrm{mg} / \mathrm{d}\end{array}$ & $\begin{array}{l}\text { Conventional treatment }+ \text { trimetazidine } 60 \mathrm{mg} / \mathrm{d}+ \\
\text { Shenmai injection } 2-4 \mathrm{ml} / \mathrm{d}\end{array}$ & $4 w$ & $(1)(6)$ \\
\hline
\end{tabular}

C: control group; E: experimental group; d: day; w: week; m: month; (1): effectiveness rate; (2): CK; (3): CK-MB; (4): LDH; (5): cTnl; (6): adverse reactions.

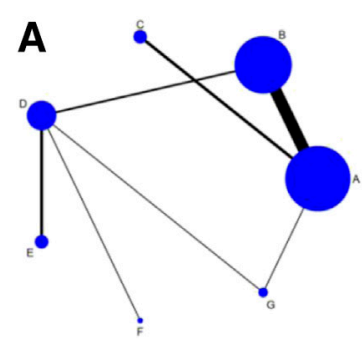

D

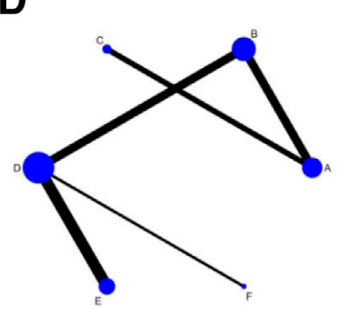

B

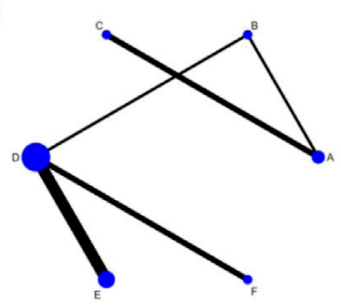

E

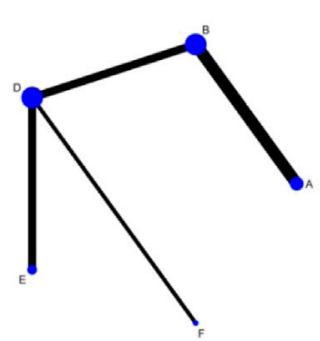

C

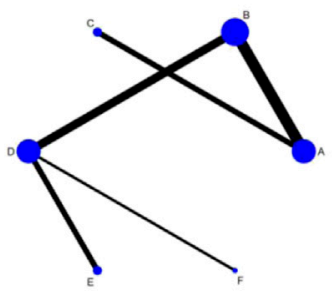

$\mathbf{F}$

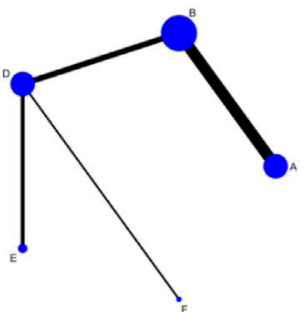

FIGURE 2 | Network diagrams of outcome indicators.(A) effectiveness rate; (B) CK; (C) CK-MB; (D) LDH; (E) cTnl; (F) adverse reaction rate. A: conventional treatment; B: conventional treatment + trimetazidine + Astragalus injection; C: conventional treatment + trimetazidine + Shenfu injection; D: conventional treatment + trimetazidine; E: conventional treatment + trimetazidine + Salviae miltiorrhizae and ligustrazine hydrochloride injection; F: conventional treatment + trimetazidine + Breviscapine injection; G: conventional treatment + trimetazidine + Shenmai injection.

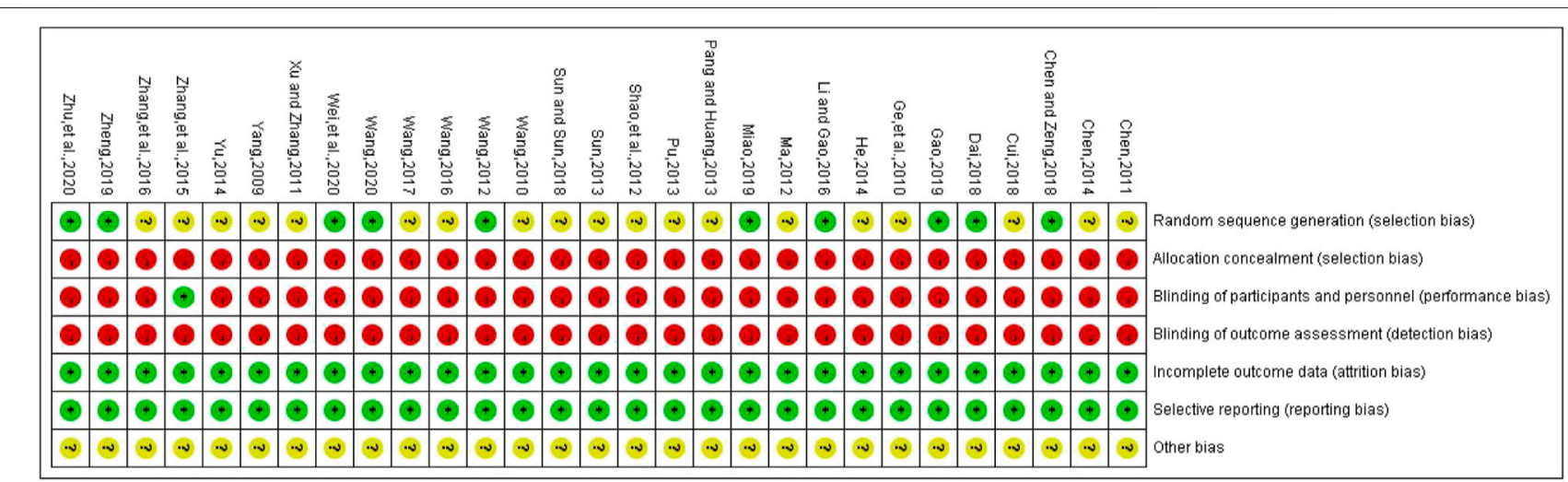

FIGURE 3 | Risk of bias for each included study. Red represents high risk of bias; green represents low risk of bias; yellow represents uncertain risk of bias.

adopted random allocation hiding and blinding to outcome evaluators, which belonged to high risk bias. One study applied blinding to patients and trial personnel, which belonged to low bias risk, while other studies did not adopt blinding, and they all belonged to high bias risk. All the data were reported completely, and there were no evidences to support the 


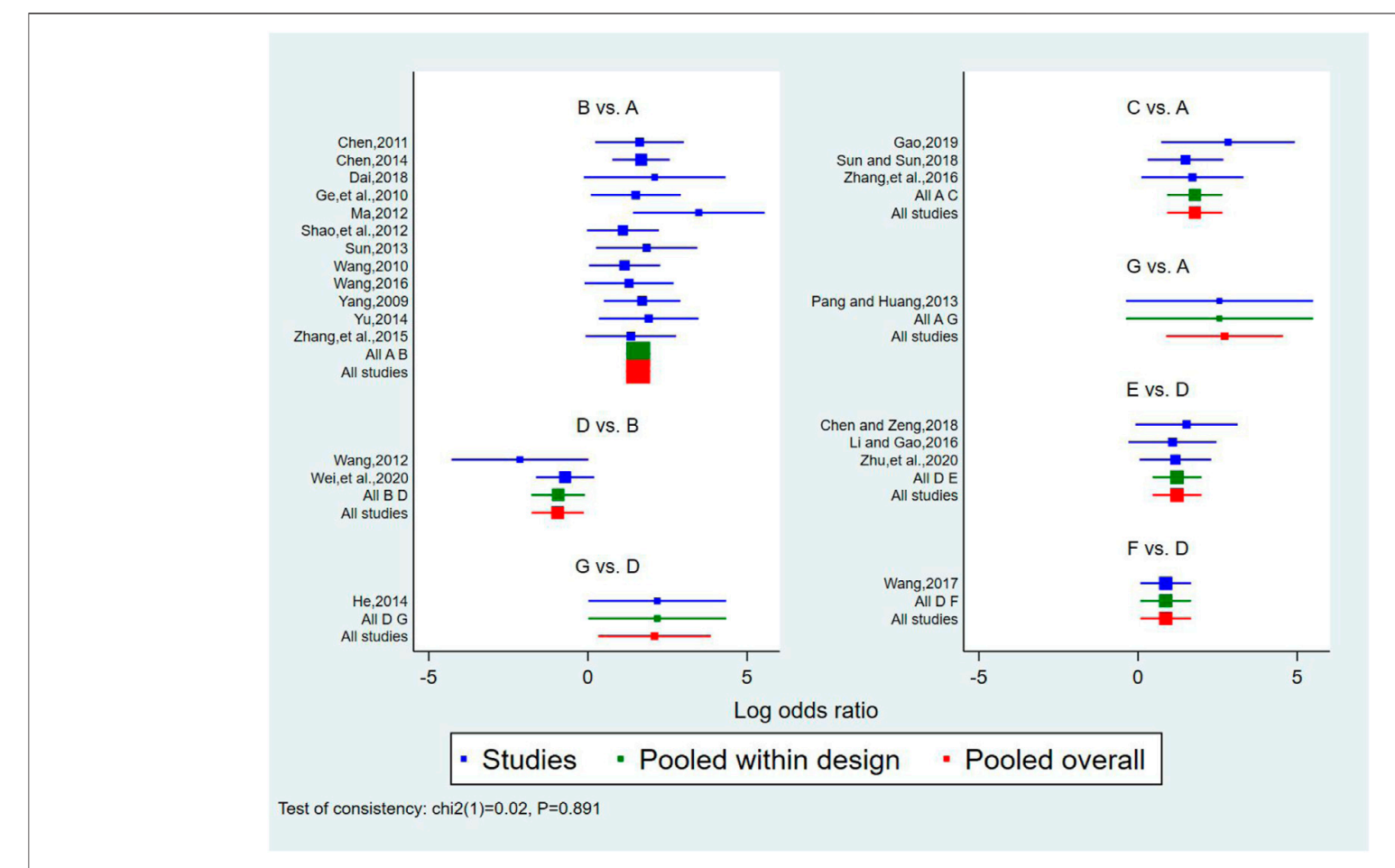

FIGURE 4 | Forest diagram of direct comparison of effectiveness rate. A: conventional treatment; B: conventional treatment + trimetazidine + Astragalus injection; C: conventional treatment + trimetazidine + Shenfu injection; D: conventional treatment + trimetazidine; E: conventional treatment + trimetazidine + Salviae miltiorrhizae and ligustrazine hydrochloride injection; F: conventional treatment + trimetazidine + Breviscapine injection; G: conventional treatment + trimetazidine + Shenmai injection.

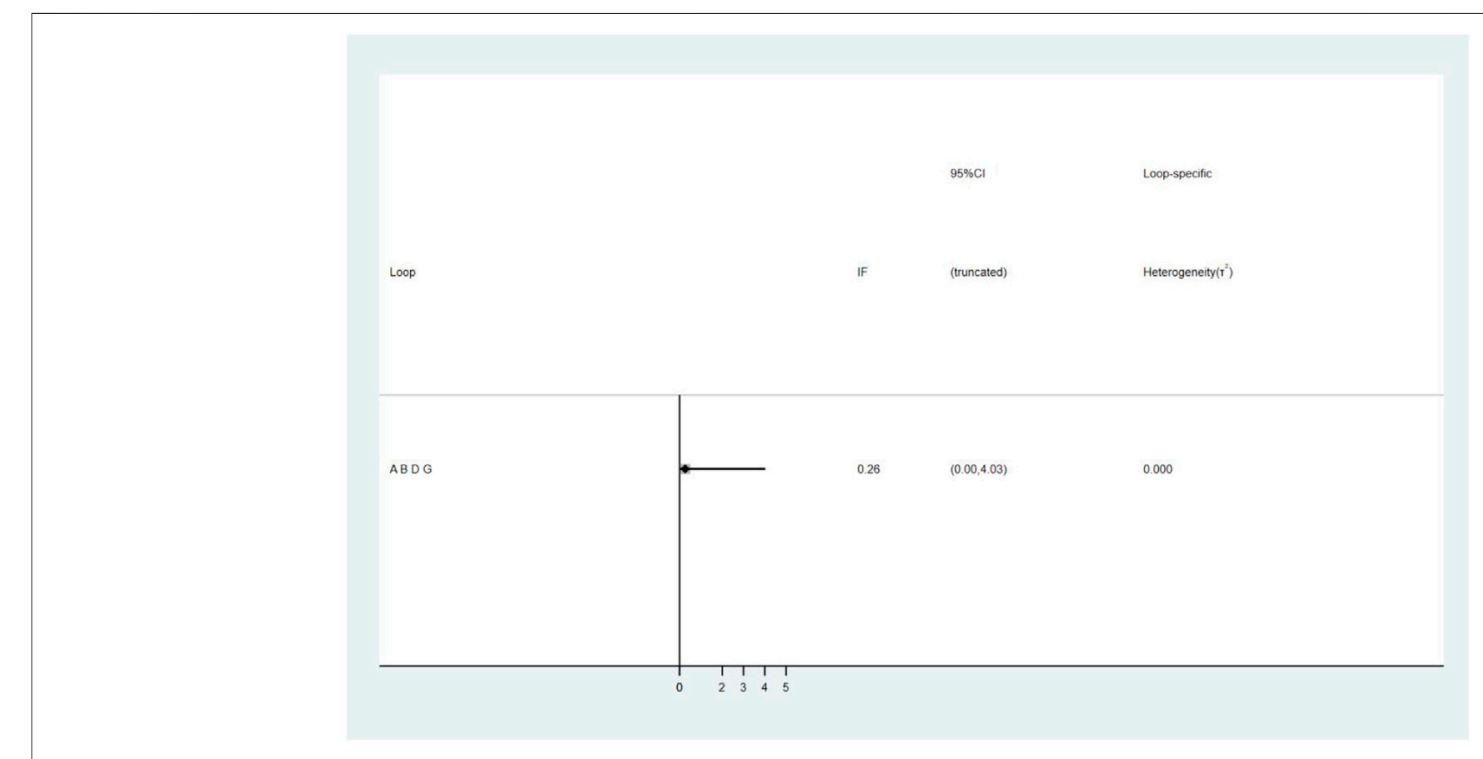

FIGURE 5 | The result of loop inconsistency test. A: conventional treatment; B: conventional treatment + trimetazidine + Astragalus injection; D: conventional treatment + trimetazidine; G: conventional treatment + trimetazidine + Shenmai injection.

selective reporting of outcomes, so it belonged to low bias risk. Whether there were other biases in all included studies could not be judged clearly, so they were at uncertain bias risk. The bias risk assessment results of included studies are detailed in Figure 3.

\section{Outcome Indicators}

\section{Effectiveness Rate}

A total of 25 studies reported effectiveness rate, of which 2 studies (Xu and Zhang, 2011; Pu, 2013) had data distortion and were not 
TABLE 2 | The network meta-analysis results of the outcome indicators.

\begin{tabular}{|c|c|c|c|c|c|c|}
\hline \multirow[t]{2}{*}{ Intervention } & \multicolumn{6}{|c|}{ Outcome indicators (OR/SMD, 95\% Cl) } \\
\hline & Effectiveness rate & CK & CK-MB & LDH & cTnl & $\begin{array}{c}\text { Adverse reaction } \\
\text { rate }\end{array}$ \\
\hline G vs. F & $3.40,(0.49,23.72)$ & - & - & - & - & - \\
\hline G vs. E & $2.38,(0.34,16.40)$ & - & - & - & - & - \\
\hline G vs. D & $8.09,(1.38,47.59)$ & - & - & - & - & - \\
\hline G vs. C & $2.54,(0.33,19.44)$ & - & - & - & - & - \\
\hline G vs. B & $3.14,(0.50,19.52)$ & - & - & - & - & - \\
\hline G vs. A & $15.17,(2.41,95.25)$ & - & - & - & - & - \\
\hline F vs. D & $2.38,(1.07,5.28)$ & $-1.90,(-3.07,-0.72)$ & $-1.68,(-4.24,0.87)$ & $-0.97,(-3.86,1.92)$ & $-3.33,(-57.22,50.56)$ & $0.23,(0.03,2.18)$ \\
\hline F vs. C & $0.75,(0.17,3.29)$ & $-1.15,(-4.17,1.88)$ & $-1.54,(-5.26 .2 .19)$ & $-0.93,(-5.21,3.35)$ & - & - \\
\hline F vs. B & $0.92,(0.29,2.89)$ & $0.21,(-1.87,2.28)$ & $1.04,(-1.93 .4 .01)$ & $0.36,(-2.98,3.70)$ & $-0.48,(-66.28,65.33)$ & $0.19,(0.02,2.14)$ \\
\hline Fvs. A & $4.46,(1.34,14.80)$ & $-2.73,(-5.51,0.05)$ & $-2.45,(-5.69 .0 .80)$ & $-1.89,(-5.64,1.87)$ & $-31.67,(-104.23,40.89)$ & $0.19,(0.02,2.34)$ \\
\hline E vs. F & $1.43,(0.47,4.32)$ & $-0.17,(-1.61,1.28)$ & $-0.62,(-3.77 .2 .52)$ & $-3.52,(-6.77,-0.27)$ & $1.08,(-64.92,67.08)$ & $6.57,(0.55,78.19)$ \\
\hline E vs. D & $3.40,(1.58,7.34)$ & $-2.06,(-2.90,-1.22)$ & $-2.31,(-4.13,-0.48)$ & $-4.49,(-5.97,-3.00)$ & $-2.25,(-40.36,35.86)$ & $1.54(0.52 .4 .53)$ \\
\hline Evs. C & $1.07,(0.25,4.64)$ & $-1.31,(-4.22,1.60)$ & $-2.16,(-5.42 .1 .10)$ & $-4.45,(-7.94,-0.96)$ & - & - \\
\hline E vs. B & $1.32,(0.43,4.06)$ & $0.04,(-1.87,1.95)$ & $0.42,(-1.95 .2 .78)$ & $-3.16,(-5.40,-0.92)$ & $0.61,(-53.04,54.25)$ & $1.28,(0.32 .5 .15)$ \\
\hline Evs. A & $6.38,(1.96,20.77)$ & $-2.90,(-5.55,-0.24)$ & $-3.07,(-5.78,-0.36)$ & $-5.40,(-8.23,-2.58)$ & $-30.59,(-92.34,31.16)$ & $1.27,(0.27 .6 .02)$ \\
\hline C vs. D & $3.18,(0.91,11.10)$ & $-0.75,(-3.54,2.04)$ & $-0.15,(-2.85 .2 .56)$ & $-0.04,(-3.20,3.12)$ & - & - \\
\hline C vs. A & $5.96,(2.50,14.23)$ & $-1.59,(-2.78,-0.40)$ & $-0.91,(-2.73 .0 .91)$ & $-0.95,(-3.01,1.10)$ & - & - \\
\hline B vs. C & $0.81,(0.31,2.10)$ & $-1.35,(-3.55,0.85)$ & $-2.58,(-0.33,-4.82)$ & $-1.29,(-3.98,1.39)$ & - & - \\
\hline B vs. D & $2.58,(1.14,5.86)$ & $-2.10,(-3.81,-0.39)$ & $-2.73,(-4.23,-1.22)$ & $-1.33,(-3.01,0.34)$ & $-2.86,(-40.76,35.05)$ & $1.21,(0.50 .2 .91)$ \\
\hline B vs. A & $4.83,(3.30,7.09)$ & $-2.94,(-4.79,-1.09)$ & $-3.49,(-4.80,-2.17)$ & $-2.25,(-3.97,-0.53)$ & $-31.19,(-62.36,-0.03)$ & $0.99,(0.50 .1 .98)$ \\
\hline A vs. D & $0.53,(0.22,1.31)$ & $0.84,(-1.68,3.36)$ & $0.76,(-1.24 .2 .76)$ & $0.92,(-1.49,3.32)$ & $28.34,(-20.43,77.11)$ & $1.21,(0.40 .3 .72)$ \\
\hline
\end{tabular}

OR, odds ratio; SMD, standardized mean difference; $C l$, confidence interval; $A$, conventional treatment; $B$, conventional treatment + trimetazidine + Astragalus injection; $C$, conventional treatment + trimetazidine + Shenfu injection; $D$, conventional treatment + trimetazidine; E, conventional treatment + trimetazidine + Salviae miltiorrhizae and ligustrazine hydrochloride injection; F, conventional treatment + trimetazidine + Breviscapine injection; $G$, conventional treatment + trimetazidine + Shenmai injection.

combined in the analysis. The remaining 23 studies constituted 7 pairs of direct comparisons, which involved 5 types of CHIs. Four types of intervention measures (A and $\mathrm{G}$ ) formed a quadrilateral closed loop, as shown in Figure 2A. The overall inconsistency test result had a $p$ value $=0.891$, indicating that the inconsistency was not significant, as shown in Figure 4. The inconsistency test of the loop resulted in an $\mathrm{IF}=0.26$ and $95 \% \mathrm{CI}(0.00,4.03)$, indicating that the inconsistency between the direct and indirect evidence was not significant, as shown in Figure 5. The consistent model was therefore adopted for network meta-analysis.

A total of 21 pairs were compared among the 7 intervention measures, 9 of which showed statistically significant differences $(p<0.05)$. On the basis of conventional treatment, combining with Astragalus injection and trimetazidine $[\mathrm{OR}=4.83,95 \% \mathrm{CI}$ $(3.30,7.09)]$, or Shenfu injection and trimetazidine [OR $=5.96$, 95\% CI $(2.50,14.23)]$, or Salviae miltiorrhizae and ligustrazine hydrochloride injection and trimetazidine $[\mathrm{OR}=6.38,95 \% \mathrm{CI}$ $(1.96,20.77)]$, or Breviscapine injection and trimetazidine [OR = 4.46 , 95\% CI $(1.34,14.80)]$, or Shenmai injection and trimetazidine $[\mathrm{OR}=15.17,95 \% \mathrm{CI}(2.41,95.25)]$ was superior to conventional treatment alone with respect to effectiveness rate. In addition to the Shenfu injection, on the basis of conventional treatment plus trimetazidine, combining with Astragalus injection $[\mathrm{OR}=2.58,95 \% \mathrm{CI}(1.14,5.86)]$, or Salviae miltiorrhizae and ligustrazine hydrochloride injection $[\mathrm{OR}=$ $3.40,95 \% \mathrm{CI}(1.58,7.34)]$ or Breviscapine injection $[\mathrm{OR}=2.38$, $95 \%$ CI $(1.07,5.28)]$, or Shenmai injection $[(\mathrm{OR}=8.09,95 \% \mathrm{CI}$ $(1.38,47.59)]$ was superior to conventional treatment plus trimetazidine in terms of effectiveness rate. The comparisons among the 5 types of injections and between conventional treatment and conventional treatment plus trimetazidine showed no statistically significant difference $(p>0.05)$. The above results are detailed in Table 2 .

Ranking the 5 types of injections according to the SUCRA resulted in the following hierarchy: Shenmai injection (89.2\%), Salviae miltiorrhizae and ligustrazine hydrochloride injection (69.1\%), Shenfu injection (65.5\%), Astragalus injection (55.2\%), and Breviscapine injection (52.3\%), as shown in Figure 6A.

\section{Creatine Kinase}

A total of 10 studies reported the level of CK, which constituted 5 pairs of direct comparisons, involved 4 types of CHIs and 6 types of intervention measures. The network diagram formed is shown in Figure 2B. Since it did not form a closed loop, no inconsistency test was carried out.

A total of 15 pairs were compared among the 6 intervention measures, 6 of which showed statistically significant differences ( $p>0.05$ ). On the basis of conventional treatment, combining with Astragalus injection and trimetazidine [SMD $=-2.94,95 \%$ CI $(-4.79,-1.09)]$, or Shenfu injection and trimetazidine [SMD = $-1.59,95 \%$ CI $(-2.78,-0.40)]$, or Salviae miltiorrhizae and ligustrazine hydrochloride injection and trimetazidine [SMD = $-2.90,95 \%$ CI $(-5.55,-0.24)]$ was superior to conventional treatment alone on reducing the level of CK. On the basis of conventional treatment plus trimetazidine, combining with Astragalus injection [SMD $=-2.10,95 \%$ CI $(-3.81,-0.39)$ ], or Salviae miltiorrhizae and ligustrazine hydrochloride injection 
A

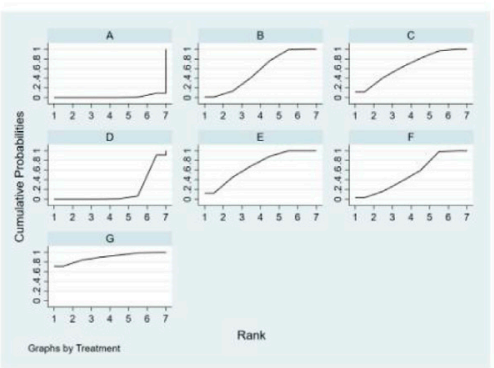

D

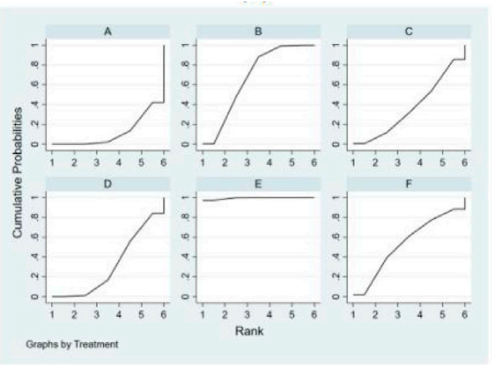

B

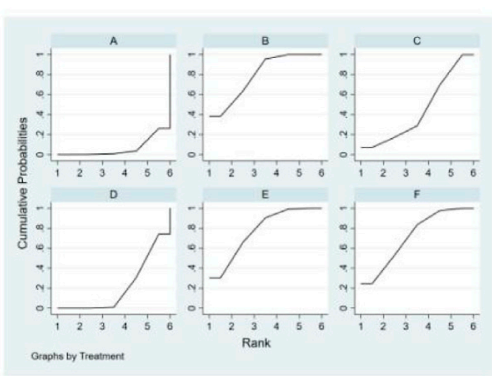

E

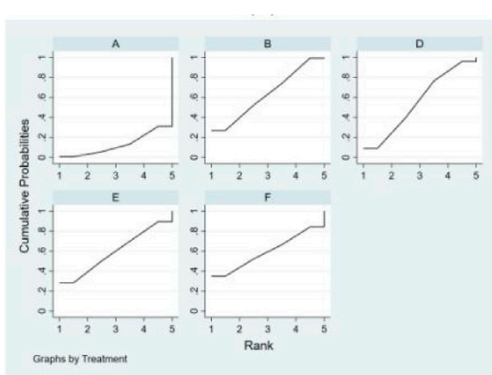

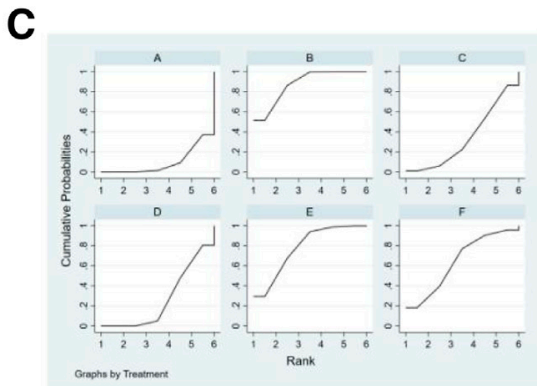

$\mathbf{F}$

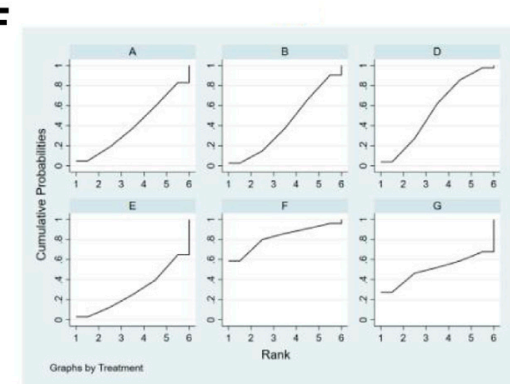

FIGURE 6 | SUCRA of outcome indicators.(A):effectiveness rate; (B):CK; (C): CK-MB; (D): LDH; (E): cTnl; (F): adverse reaction rate. A: conventional treatment; B: conventional treatment + trimetazidine + Astragalus injection; C: conventional treatment + trimetazidine + Shenfu injection; D: conventional treatment + trimetazidine; E: conventional treatment + trimetazidine + Salviae miltiorrhizae and ligustrazine hydrochloride injection; F: conventional treatment + trimetazidine + Breviscapine injection; G: conventional + treatment + trimetazidine + Shenmai injection

$[\mathrm{SMD}=-2.06,95 \% \mathrm{CI}(-2.90,-1.22)]$ or Breviscapine injection $[\mathrm{SMD}=-1.90,95 \% \mathrm{CI}(-3.07,-0.72)]$ was superior to conventional treatment plus trimetazidine on reducing the level of CK. The comparisons among the 4 types of injections and between conventional treatment and conventional treatment plus trimetazidine showed no statistically significant difference $(p>0.05)$. The above results are detailed in Table 2.

Ranking the 4 injections based on the SUCRA value, the results were as follows: Astragalus injection (79.5\%), Salviae miltiorrhizae and ligustrazine hydrochloride injection (77.1\%), Breviscapine injection (71.7\%), and Shenfu injection (44.5\%), as shown in Figure 6B.

\section{Creatine Kinase-MB}

A total of 12 studies reported the level of CK-MB, which constituted 5 pairs of direct comparisons, involved 4 types of CHIs and 6 types of intervention measures. The network diagram formed is shown in Figure 2C. It did not form a closed loop, so there was no inconsistency test.

A total of 15 pairs of comparisons were formed among the 6 intervention measures, 5 of which were statistically significant $(p<0.05)$. On the basis of conventional treatment, combining with Astragalus injection and trimetazidine [SMD $=-3.49,95 \%$ CI $(-4.80,-2.17)]$, or Salviae miltiorrhizae and ligustrazine hydrochloride injection and trimetazidine [SMD $=-3.07,95 \%$ CI $(-5.78,-0.36)]$ was superior to conventional treatment alone on reducing the level of CK-MB. On the basis of conventional treatment plus trimetazidine, combining with Astragalus injection $[\mathrm{SMD}=-2.73,95 \% \mathrm{CI}(-4.23,-1.22)]$, or Salviae miltiorrhizae and ligustrazine hydrochloride injection [SMD = $-2.31,95 \%$ CI $(-4.13,-0.48)]$ was superior to conventional treatment plus trimetazidine on reducing the level of CK-MB. The comparisons among the 4 types of injections showed that on the basis of conventional treatment plus trimetazidine, combining with Astragalus injection was superior to Shenfu injection $[\mathrm{SMD}=-2.58,95 \% \mathrm{CI}(-0.33,-4.82)]$ on reducing the level of CK-MB. The other 10 pair comparisons showed no statistically significant difference $(p>0.05)$. The above results are detailed in Table 2.

Based on the SUCRA value, the 4 types of injections were ranked as follows: Astragalus injection (87.5\%), Salviae miltiorrhizae and ligustrazine hydrochloride injection (77.9\%), Breviscapine injection (64.3\%), and Shenfu injection (33.9\%), as shown in Figure 6C.

\section{Lactate Dehydrogenase}

A total of 13 studies reported the level of $\mathrm{LDH}$, which constituted 5 pairs of direct comparisons, involved 4 types of CHIs and 6 types of intervention measures. The network diagram formed is shown in Figure 2D. No closed loop was constituted and no inconsistency test was performed.

A total of 15 pairs of comparisons were formed among the 6 intervention measures, 6 of which were statistically significant $(p<0.05)$. On the basis of conventional treatment, combining with Astragalus injection and trimetazidine [SMD $=-2.25,95 \%$ CI $(-3.97,-0.53)]$, or Salviae miltiorrhizae and ligustrazine hydrochloride injection and trimetazidine [SMD $=-5.40,95 \%$ 
A

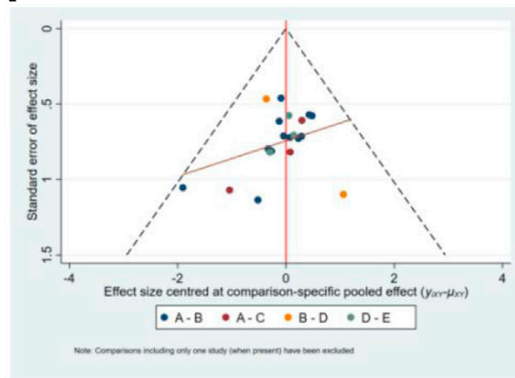

D

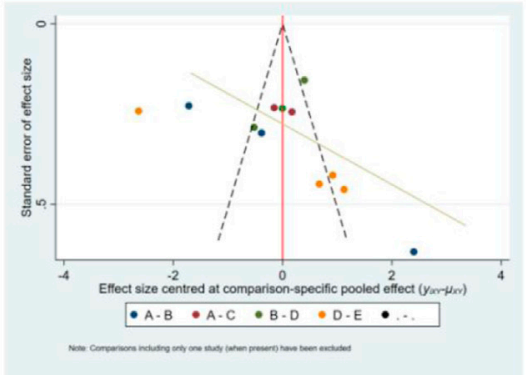

B

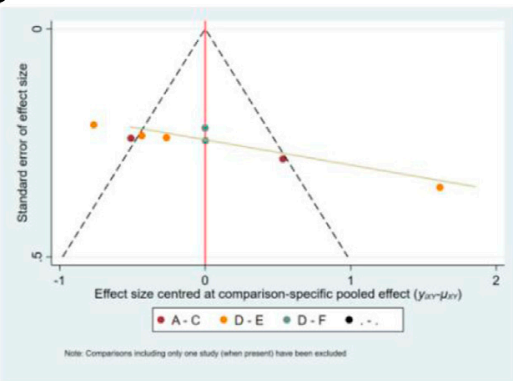

E

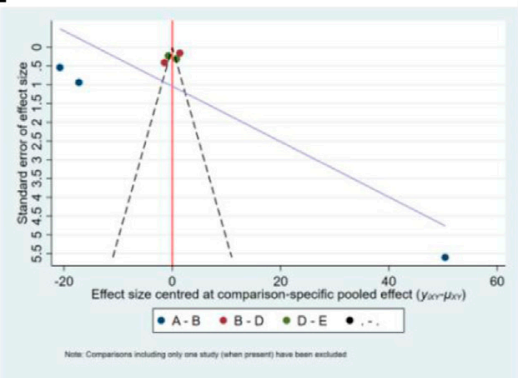

C

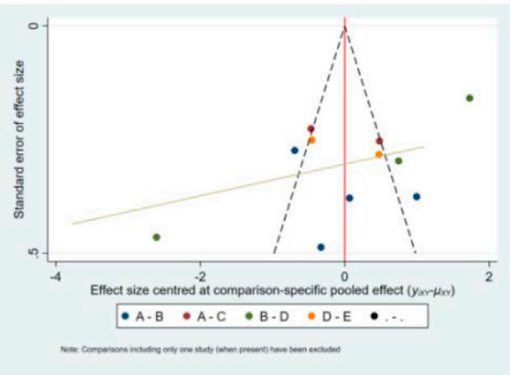

$\mathbf{F}$

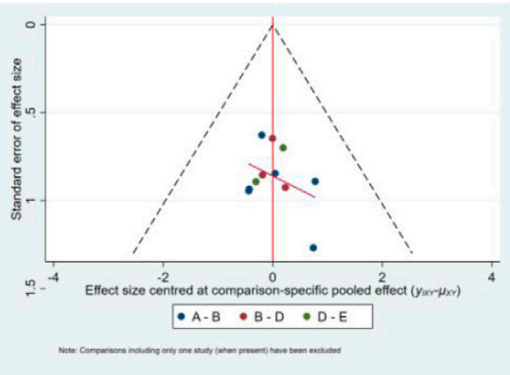

FIGURE 7 | The funnel plot of outcome indicators. A:effectiveness rate; B:CK; C:CK-MB; $\mathbf{D}: \mathrm{LDH}$; E:cTnl; F:adverse reaction rate. A: conventional treatment; B: conventional treatment + trimetazidine + Astragalus injection; C: conventional treatment + trimetazidine + Shenfu injection; D: conventional treatment + trimetazidine; E: conventional treatment + trimetazidine + Salviae miltiorrhizae and ligustrazine hydrochloride injection; F: conventional treatment + trimetazidine + Beviscapine injection.

CI $(-8.23,-2.58)]$ was superior to conventional treatment alone on reducing the level of $\mathrm{LDH}$. On the basis of conventional treatment plus trimetazidine, combining with Salviae miltiorrhizae and ligustrazine hydrochloride injection [SMD = -4.49 , 95\% CI $(-5.97,-3.00)]$ was superior to conventional treatment plus trimetazidine on reducing the level of $\mathrm{LDH}$. The comparisons among the 4 types of injections showed that on the basis of conventional treatment plus trimetazidine, combining with Salviae miltiorrhizae and ligustrazine hydrochloride injection was superior to Astragalus injection $[\mathrm{SMD}=-3.16,95 \% \mathrm{CI}(-5.40,-0.92)]$ or Shenfu injection $[\mathrm{SMD}=-4.45,95 \% \mathrm{CI}(-7.94,-0.96)]$ or Breviscapine injection $[\mathrm{SMD}=-3.52,95 \% \mathrm{CI}(-6.77,-0.27)]$ on reducing LDH. The other 9 pairs comparisons were not statistically significant $(p>0.05)$. The above results are detailed in Table 2 .

Ranking the 4 injections based on the SUCRA value, the results were as follows: Salviae miltiorrhizae and ligustrazine hydrochloride injection (99.4\%), Astragalus injection (66.7\%), Breviscapine injection (54.3\%), and Shenfu injection (36.4\%), as shown in Figure 6D.

\section{Cardiac Troponin I}

A total of 8 studies reported the level of cTnI, which constituted 4 pairs of direct comparisons, involved 3 types of CHIs and 5 types of intervention measures. The network diagram formed is shown in Figure 2E. Since it did not constitute a closed loop, the inconsistency test was not performed.

The results showed that there were no statistically significant differences among 10 pairs of comparisons of the
5 intervention measures $(p>0.05)$. The above results are detailed in Table 2.

Based on the SUCRA value, the 3 injections were ranked as follows: Astragalus injection (63.2\%), Salviae miltiorrhizae and ligustrazine hydrochloride injection (59.4\%), and Breviscapine injection (59.4\%), as shown in Figure 6E.

\section{Adverse Reaction Rate}

Eight studies reported no adverse reactions during treatment, and 12 studies reported the number of cases of adverse reactions. The adverse reactions mainly include dizziness, headache, abdominal discomfort, nausea and vomiting, poor appetite, diarrhea, and rash. In terms of the adverse reaction rate, 12 studies consisted 4 pairs of comparisons, involved 3 types of CHIs and 5 types of intervention measures. The network diagram formed is shown in Figure 2F. Since it did not constitute a closed loop, the inconsistency test was not performed. The results showed that there were no statistically significant differences among 10 pairs comparisons of the 5 intervention measures $(p>0.05)$. The above results are detailed in Table 2.

Ranking the three injections based on the SUCRA value, the results were as follows: Breviscapine injection (90.4\%), Astragalus injection (39.3\%), and Salviae miltiorrhizae and ligustrazine hydrochloride injection (26.1\%), as shown in Figure 6F.

\section{Assessment of Publication Bias}

The funnel plots have poor symmetry, indicating that there was some publication bias in the included studies, which may be caused by small sample effects, as shown in Figure 7 . 


\section{DISCUSSION}

In the present study, we conducted a search of the RCTs of CHIs combined with trimetazidine in the treatment of VMC, and adopted a network meta-analysis approach to evaluate the clinical efficacy and safety of different CHIs combined with trimetazidine. A total of 29 RCTs were included in this analysis, representing 7 types of intervention measures and 5 types of CHIs. Our findings indicate that, in terms of effectiveness rate and reduction of the $\mathrm{CK}$ and $\mathrm{CK}-\mathrm{MB}$ levels, Astragalus injection or Salviae miltiorrhizae and ligustrazine hydrochloride injection combined with trimetazidine was superior to conventional treatment alone or conventional treatment combined with trimetazidine. Moreover, Salviae miltiorrhizae and ligustrazine hydrochloride injection combined with trimetazidine was also superior to conventional treatment or conventional treatment combined with trimetazidine in reducing the level of $\mathrm{LDH}$, and Astragalus injection combined with trimetazidine was also superior to conventional treatment in reducing the level of $\mathrm{LDH}$. Shenmai injection or Breviscapine injection combined with trimetazidine was better than conventional treatment or conventional treatment combined with trimetazidine in terms of effectiveness rate. Breviscapine injection combined with trimetazidine was superior to conventional treatment combined with trimetazidine in reducing the level of $\mathrm{CK}$. Shenfu injection combined with trimetazidine was superior to conventional treatment alone in terms of effectiveness rate and reduction of CK level. The comparison of different CHIs showed that Astragalus injection was superior to Shenfu injection in reducing the level of CK-MB. In reducing the level of $\mathrm{LDH}$, Salviae miltiorrhizae and ligustrazine hydrochloride injection was superior to Astragalus injection, Shenfu injection, and Breviscapine injection. In terms of safety, there was no significant difference among different interventions. Based on the ranking of the SUCRA value, Shenmai injection may be superior to other injections in terms of effectiveness rate, Astragalus injection and Salviae miltiorrhizae and ligustrazine hydrochloride injection may be superior to other injections in reducing myocardial injury markers. However, different CHIs have different properties. Some tend to replenish qi, some tend to activate blood circulation, some tend to tonify yang, and some tend to nourish yin. Therefore, for the selection of CHI, it is better to choose based on the performance of patients and syndrome differentiation of traditional Chinese medicine in clinical practice, which may be able to harvest a better clinical efficacy.

As a myocardial metabolic agent, trimetazidine can optimize the oxidation of fatty acids and glucose, improve myocardial metabolism, inhibit cardiomyocyte apoptosis, reduce cardiac remodeling, improve cardiac function and so on, which is often used in the treatment of cardiovascular diseases ( $\mathrm{Li}$ et al., 2020). Astragalus injection is extracted from the root of Astragalus mongholicus Bunge. Previous studies have shown that the application of Astragali Radix can improve the survival rate and relieve the pathological changes of mice with VMC induced by coxsackievirus B3 (Chen et al., 2006). Astragaloside IV plays a cardioprotective role in experimental animals with VMC through a variety of signal pathways, such as antimyocardial remodeling, antivirus, anti-oxidation, anti-inflammation, anti-apoptosis, and reducing myocardial fibrosis (Zhuang et al., 2019). Salviae miltiorrhizae and ligustrazine hydrochloride injection is a compound preparation composed of ligustrazine hydrochloride and the extract of Salvia miltiorrhiza Bunge. Salviae miltiorrhizae Radix et Rhizoma is a standard Chinese herbal medicine for promoting blood circulation and removing blood stasis, and is thus widely used in the treatment of cardiovascular diseases. Previous studies have shown that several active components of Salviae miltiorrhizae Radix et Rhizoma have significant antiinflammatory and antioxidant activities (MEIm et al., 2019). Tanshinone IIA can reduce myocardial apoptosis and myocardial remodeling caused by overload (Feng et al., 2016) and has been shown to alleviate cardiac dysfunction in septic mice by inhibiting inflammatory response (Huang et al., 2015). Tanshinol enhances antioxidant activity by activating serine/ threonine kinase/extracellular signaling-regulated kinase1/2/ nuclear factor erythroid-2-related factor 2 (Akt/ERK1/2/Nrf2) signal pathway, thus exerting a cardioprotective function ( $\mathrm{Yu}$ et al., 2015). Ligustrazine hydrochloride can reduce cardiomyocyte apoptosis and injury in coxsackievirus B3-induced VMC by downregulating the expression of membrane surface molecules in mouse cardiomyocytes (Jiang et al., 2014). Breviscapine injection, which is extracted from Erigeron breviscapus (Vaniot.) Hand-Mazz., has been shown in previous studies to have pharmacological effects, such as antiinflammation, endothelial protection, myocardial protection, and reduction of cardiac remodeling, leading to its widespread use in the treatment of cardiovascular disease (Gao et al., 2017). Shenmai injection is derived from Panax ginseng C. A. Mey. and Ophiopogon japonicus (Thunb.) Ker Gawl., and has been shown in systematic reviews to be of use in the treatment of VMC (Lu et al., 2014). Ginsenoside Rb3, an active components of Ginseng Radix et Rhizoma, can inhibit endothelial to mesenchymal transformation of cardiac microvascular cells following coxsackievirus B3 infection through protein-rich tyrosine kinase 2-phosphoinositide-3-kinase/ serine/threonine kinase (Pyk2-PI3K-AKT) signal pathway (Yang et al., 2019). Finallly, Shenfu injection is derived from Panax ginseng C. A. Mey and Aconitum carmichaeli Debeaux, and is widely used in China for the treatment of acute and critical cardiovascular diseases. Studies have shown that Shenfu injection may play a role in antiviral myocarditis by regulating multiple metabolic pathways, such as sphingolipid metabolism, glycerophospholipid metabolism, arachidonic acid metabolism, tryptophan metabolism, amino acyl RNA biosynthesis, and the citrate cycle (Tan et al., 2018).

Although the present study compared the efficacy and safety of different types of CHIs combined with trimetazidine in the treatment of VMC by network meta-analysis and provided some reference for the selection of $\mathrm{CHI}$ in clinic, there are still some limitations in this study. First, the methodological quality of the included studies was generally low. The method of generating random sequences in most studies is not clear, and all studies have no random allocation hiding, which may lead to selective bias in the determination of subjects. Almost all the included studies have not blinded the patients, trial personnel, and outcome evaluator, which may lead to expectation bias due 
to the influence of subjective factors in the evaluation of outcome indicators. Second, there may be some clinical heterogeneity because of some differences in the age of participators, drug dosage, and course of treatment in the included studies. Some studies do not monitor safety, leading to the failure to assess the safety of some CHIs. In addition, there may be a small sample effect, resulting in some publication bias in the included study. In view of the above limitations, we suggest that more high-quality, large-sample, standardized clinical randomized controlled trials should be carried out in the future, to provide strong evidence for the efficacy and safety of CHIs combined with trimetazidine for the treatment of VMC.

\section{CONCLUSION}

The results of our network meta-analysis showed that $\mathrm{CHI}$ combined with trimetazidine may have therapeutic effect in the treatment of VMC. Compared with conventional treatment alone or conventional treatment combined with trimetazidine, the clinical effectiveness rate of $\mathrm{CHI}$ combined with trimetazidine is higher, with a greater effect on reducing myocardial zymogram level and no significant difference in safety. Among these CHIs evaluated in our analysis, Shenmai injection, Astragalus injection, and Salviae miltiorrhizae and ligustrazine hydrochloride injection may be the most effective. Given the limitations in the design of the included studies, our conclusions require further verification in larger, multicenter, and randomized controlled trials.

\section{REFERENCES}

Cao, Y., Xu, X., and Zhang, P. (2015). Advances in the traditional Chinese medicine-based management of viral myocarditis. Cell Biochem. Biophys. 73 (1), 237-243. doi:10.1007/s12013-015-0620-x

Chen, C. L. (2011). 32 cases of acute viral myocarditis with the treatment of traditional Chinese medicine combined with western medicine. China's Naturopathy 19 (07), 57. doi:10.19621/j.cnki.11-3555/r.2011.07.059

Chen, P. G. (2014). Efficacy of trimetazidine combined with Astragalus injection in the treatment of viral acute myocarditis. Chin. Foreign Med. Res. 12 (01), 105-106. doi:10.14033/j.cnki.cfmr.2014.01.048

Chen, X.-J., Bian, Z.-P., Lu, S., Xu, J.-D., Gu, C.-R., Yang, D., et al. (2006). Cardiac protective effect of Astragalus on viral myocarditis mice: comparison with perindopril. Am. J. Chin. Med. 34 (3), 493-502. doi:10.1142/s0192415x06004028

Chen, X. H., and Zeng, Y. S. (2018). Efficacy of Salviae Miltiorrhizae and Ligustrazine hydrochloride injection combined with trimetazidine in the treatment of VMC in acute stage. Shenzhen J. Integrated Traditional Chin. West. Med. 28 (21), 36-37. doi:10.16458/j.cnki.1007-0893.2018.21.016

Cheng, L. X. (2015). Efficacy of trimetazidine combined with Shenmai injection in the treatment of acute viral myocarditis. Cardiovasc. Dis. J. integrated traditional Chin. West. Med. 3 (27), 36-37. doi:10.16282/j.cnki.cn11-9336/r.2015.27.021

Cui, S. Q. (2018). Effect of trimetazidine combined with Astragalus injection on resisting inflammation and oxygen radicals in patients with acute viral myocarditis. J. Hainan Med. Univ. 24 (07), 337-739. doi:10.13210/j.cnki.jhmu.20180322.001

Dai, M. M. (2018). Analysis of the Efficacy of trimetazidine combined with Astragalus injection in the treatment of myocarditis. China Foreign Med. Treat. 37 (28), 110-112. doi:10.16662/j.cnki.1674-0742.2018.28.110

Feng, J., Li, S., and Chen, H. (2016). Tanshinone IIA inhibits myocardial remodeling induced by pressure overload via suppressing oxidative stress

\section{AUTHOR CONTRIBUTIONS}

Conception and design: DW and XY. Literature retrieval, data extraction, and analysis: KW, BY, ZH, LH, and YH. Interpretation of results: DD and KW. Manuscript writing: $\mathrm{KW}, \mathrm{DD}$, and $\mathrm{BY}$.

\section{FUNDING}

This work is supported by The High-Level Key Disciplines Project of Guangzhou University of Chinese Medicine (No. A1-AFD018171Z11089 to DW) and Scientific Research Project of Traditional Chinese Medicine Bureau of Guangdong Province (NO:20184015).

\section{ACKNOWLEDGMENTS}

We thank Clare Cox, from Liwen Bianji, Edanz Editing China (www.liwenbianji.cn/ac), for editing the English text of the draft of this manuscript.

\section{SUPPLEMENTARY MATERIAL}

The Supplementary Material for this article can be found online at: https://www.frontiersin.org/articles/10.3389/fphar.2021.630896/ full\#supplementary-material.

and inflammation: possible role of silent information regulator 1. Eur. J. Pharmacol. 791, 632-639. doi:10.1016/j.ejphar.2016.09.041

Gao, J., Chen, G., He, H., Liu, C., Xiong, X., Li, J., et al. (2017). Therapeutic effects of Breviscapine in cardiovascular diseases: a review. Front. Pharmacol. 8, 289. doi:10.3389/fphar.2017.00289

Gao, Y. (2019). Efficacy evaluation of trimetazidine combined with Shenfu injection in the treatment of viral myocarditis. Modern Digestion and Intervention (A02) 24, 2120-2121.

Ge, H. Z., Xu, Y., and Qin, H. Y. (2010). Clinical observation of Astragalus injection combined with trimetazidine in treating 60 cases of acute viral myocarditis. HeiLong Jiang Med. J. 34 (2), 134-136. doi:10.3969/j.issn.1004-5775.2010.02.023

Global Burden of Disease Study 2013 Collaborators (2015). Global, regional, and national incidence, prevalence, and years lived with disability for 301 acute and chronic diseases and injuries in 188 countries, 1990-2013: a systematic analysis for the Global Burden of Disease Study 2013. Lancet 386 (9995), 743-800. doi:10.1016/S0140-6736(15)60692-4

Grün, S., Schumm, J., Greulich, S., Wagner, A., Schneider, S., Bruder, O., et al. (2012). Long-term follow-up of biopsy-proven viral myocarditis. J. Am. Coll. Cardiol. 59 (18), 1604-1615. doi:10.1016/j.jacc.2012.01.007

He, L. (2014). Clinical observation of trimetazidine combined with Shenmai injection in the treatment of viral myocarditis. China Health Care and Nutrition 24 (4), 2249. doi:10. 3969/j.issn.1004-7484(x).2014.04.559

Higgins, J. P. T., Altman, D. G., Gotzsche, P. C., Jüni, P., Moher, D., Oxman, A. D., et al. (2011). Cochrane bias methods group, and Cochrane statistical methods GroupThe Cochrane collaboration's tool for assessing risk of bias in randomised trials. Bmj 343, d5928. doi:10.1136/bmj.d5928

Huang, L., Zheng, M., Zhou, Y., Zhu, J., Zhu, M., Zhao, F., et al. (2015). Tanshinone IIA attenuates cardiac dysfunction in endotoxin-induced septic mice via inhibition of NADPH oxidase 2-related signaling pathway. Int. Immunopharmacol. 28 (1), 444-449. doi:10.1016/j.intimp.2015.07.004 
Jiang, H., Chen, X. X., and Wang, J. Y. (2014). Effects of Ligustrazine hydrochloride on cardiomyocyte apoptosis in mice infected with Coxsackie virus B3. Chin. J. Nosocomiol. 24 (13), 3121-3123. doi:10.11816/cn.ni.2014-134233

Leone, O., Pieroni, M., Rapezzi, C., and Olivotto, I. (2019). The spectrum of myocarditis: from pathology to the clinics. Virchows Arch. 475 (3), 279-301. doi:10.1007/s00428-019-02615-8

Li, H., Ma, Z., Zhai, Y., Lv, C., Yuan, P., Zhu, F., et al. (2020). Trimetazidine ameliorates myocardial metabolic remodeling in isoproterenol-induced rats through regulating ketone body metabolism via activating AMPK and PPAR $\alpha$. Front. Pharmacol. 11, 1255. doi:10.3389/fphar.2020.01255

Li, J. P., Liu, Y., Guo, J. M., Shang, E. X., Zhu, Z. H., Zhu, K. Y., et al. (2017). A comprehensive strategy to evaluate compatible stability of Chinese medicine injection and infusion solutions based on chemical analysis and bioactivity assay. Front. Pharmacol. 8, 833. doi:10.3389/fphar.2017.00833

Li, Q., and Gao, X. F. (2016). Clinical observation of Salviae Miltiorrhizae and Ligustrazine hydrochloride injection combined with trimetazidine in the treatment of acute viral myocarditis. China Pharm. 27 (32), 4568-4570. doi:10.6039/j.issn.1001-0408.2016.32.34

Liu, S., Niu, H., and Zhang, J. G. (2015). Meta-analysis of the efficacy and safety of Astragalus injection combined with trimetazidine in the treatment of viral myocarditis. China Pharmary 26 (36), 5113-5116. doi:10.6039/j.issn.1001-0408.2015.27.26

Lu, L. Y., Zheng, G. Q., and Wang, Y. (2014). An overview of systematic reviews of Shenmai injection for healthcare. Evid. Based Complement. Alternat. Med. 2014, 1-9. doi:10.1155/2014/840650

$\mathrm{Ma}, \mathrm{Z}$. X. (2012). Clinical observation on 62 cases of viral myocarditis treated by trimetazidine combined with Astragalus injection. Chin. J. Coal Industry Med. 15 (03), 380-381.

Marzilli, M., Vinereanu, D., Lopaschuk, G., Chen, Y., Dalal, J. J., Danchin, N., et al. (2019). Trimetazidine in cardiovascular medicine. Int. J. Cardiol. 293, 39-44. doi:10.1016/j.ijcard.2019.05.063

MEIm, X.-D., Cao, Y.-F., Che, Y.-Y., Li, J., Shang, Z.-P., Zhao, W.-J., et al. (2019). Danshen: a phytochemical and pharmacological overview. Chin. J. Nat. Med. 17 (1), 59-80. doi:10.1016/s1875-5364(19)30010-x

Miao, Y. J. (2019). Effects of Breviscapine injection combined with trimetazidine on the improvement of symptoms and changes of myocardial enzyme levels in patients with acute viral myocarditis. Capital Med. 26 (07), 49. doi:10.3969/j. issn.1005-8257.2019.07.041

Olejniczak, M., Schwartz, M., Webber, E., Shaffer, A., and Perry, T. E. (2020). Viral myocarditis-incidence, diagnosis and management. J. Cardiothorac. Vasc. Anesth. 34 (6), 1591-1601. doi:10.1053/j.jvca.2019.12.052

Pang, M. X., and Huang, Z. (2013). Clinical observation of trimetazidine combined with Shenmai injection in the treatment of viral myocarditis in children. J. Youjiang Med. Coll. Nationalities 68 (2), 207-208. doi:10.3969/j.issn.1001-5817.2013.02.052

$\mathrm{Pu}, \mathrm{X} . \mathrm{H}$. (2013). Analysis of the clinical effect of trimetazidine combined with Astragalus injection in the treatment of 134 cases of myocarditis. China Health Industry 10 (06), 69. doi:10.16659/j.cnki.1672-5654.2013.06.094

Shao, L. L., Han, L. Y., Xin, S. L., Chang, C., Li, Z. M., and Liu, L. J. (2012). Analysis of trimetazidine combined with Astragalus injection in the treatment of myocarditis. Zhejiang Clin. Med. J. 14 (09), 1106-1107. doi:10.3969/j.issn.1008-7664.2012.09.040

Society of Cardiovascular Diseases, China Association of Chinese Medicine (2020). International clinical practice guideline of traditional Chinese medicine-viral myocarditis. Chin. J. Exp. Traditional Med. Formulae 26 (18), 91-97. doi:10. 13422/j.cnki.syfjx.20201062

Sun, X. P. (2013). Clinical observation of trimetazidine and Astragalus in the treatment of acute viral myocarditis. Contemp. Med. 19 (07), 148-149. doi:10. 3969/j.issn.1009-4393.2013.7.113

Sun, X., and Sun, J. L. (2018). Efficacy of trimetazidine combined with Shenfu injection in the treatment of viral myocarditis. Chin. Community Doctors 34 (3), 80-81. doi:10.3969/j.issn.1007-614x.2018.3.50

Tan, G., Zhou, Q., Liu, K., Dong, X., Li, L., Liao, W., et al. (2018). Cross-platform metabolic profiling deciphering the potential targets of Shenfu injection against acute viral myocarditis in mice. J. Pharm. Biomed. Anal. 160, 1-11. doi:10.1016/ j.jpba.2018.07.042

Tschöpe, C., Cooper, L. T., Torre-Amione, G., and Van Linthout, S. (2019). Management of myocarditis-related cardiomyopathy in adults. Circ. Res. 124 (11), 1568-1583. doi:10.1161/circresaha.118.313578

Wang, J. (2017). Efficacy of trimetazidine combined with Breviscapine injection in the treatment of acute viral myocarditis. Mod. J. Integrated Traditional Chin. West. Med. 26 (8), 879-881. doi:10.3969/j.issn.1008-8849.2017.08.029
Wang, J. F. (2020). Effects of Salviae Miltiorrhizae and Ligustrazine hydrochloride combined with trimetazidine on serum lipid peroxides, IFN- $\gamma$ and myocardial enzymes in patients with viral myocarditis. Chin J. Clin. Rational Drug Use 13 (2B), 51-53. doi:10.15887/j.cnki.13-1389/r.2020.08.028

Wang, Q. (2012). Efficacy and safety of trimetazidine combined with Astragalus injection in treating acute viral myocarditis. Chin. J. Gerontol. 32 (9), 1820-1821. doi:10.3969/j.issn.1005-9202.2012.09.018

Wang, Q. J. (2016). Clinical effects and safety of trimetazidine combined with Astragalus radix injection in treating acute viral myocarditis. Chin. J. Clin. Rational Drug Use 9 (3C), 8-9. doi:10.15887/j.cnki.13-1389/r.2016.09.004

Wang, Y. C. (2019). Clinical effect of trimetazidine combined with Breviscapine injection in treating acute viral myocarditis. Mod. Diagn. Treat. 30 (24), 4320-4322.

Wang, Z. X. (2010). Clinical observation of trimetazidine combined with Astragalus injection in the treatment of myocarditis. J. China Traditional Chin. Med. Inf. 2 (28), 134.

Wei, A., Li, Q., Ding, L. X., Tan, X. H., Guo, D. M., Gu, Q., et al. (2020). Effect of Astragalus injection combined with trimetazidine on NLRP3 inflammasome and related downstream pathways in peripheral blood of elderly patients with viral myocarditis. Mod. J. Integrated Traditional Chin. West. Med. 29 (8), 815-818. doi:10.3969/j.issn.1008-8849.2020.08.005

Xu, W. M., and Zhang, S. F. (2011). Astragalus injection combined with Wanshuangli in the treatment of 60 cases of viral myocarditis. Contemp. Med. 17 (19), 39-40. doi:10.3969/j.issn.1009-4393.2011.19.021

Yang, L., Liu, Q., Yu, Y., Xu, H., Chen, S., and Shi, S. (2019). Ginsenoside-Rb3 inhibits endothelial-mesenchymal transition of cardiac microvascular endothelial cells. Herz 44 (1), 60-68. doi:10.1007/s00059-017-4628-4

Yang, S. Y. (2009). Observation on the curative effect of Astragalus injection combined with trimetazidine in the treatment of acute viral myocarditisAnalysis of 45 cases. New Med. 40 (2), 101-103.

Yu, J., Wang, L., Akinyi, M., Li, Y., Duan, Z., Zhu, Y., et al. (2015). Danshensu protects isolated heart against ischemia reperfusion injury through activation of Akt/ERK1/2/ Nrf2 signaling. Int. J. Clin. Exp. Med. 8 (9), 14793-14804.

Yu, K., Wang, Y. N., Ren, Y., Zhang, X. W., Zhang, X. F., and Zhang, H. (2014). Trimetazidine:a meta-analysis of randomized controlled trial to the patients with viral myocarditis. Chin. J. Nosocomiol 24 (24), 6139-6141. doi:10.11816/cn.ni.2014-143898

Yu, X. L. (2014). Clinical observation of Astragalus injection combined with trimetazidine for the treatment of acute viral myocarditis. Int. J. Virol. 21 (5), 226-230. doi:10.3706/cma.j.issn.1673-4092.2014.05.010

Zhang, H. X., Guan, S. C., and Zhao, L. X. (2015). Therapeutic effect of trimetazidine combined with Astragalus injection on acute viral myocarditis. Contemp. Med. 21 (13), 122-123. doi:10.3969/j.issn.1009-4393.2015.13.081

Zhang, Y. J., Fu, Y. Z., and Chen, J. D. (2016). Efficacy analysis of trimetazidine combined with Shenfu injection in treating viral myocarditis. China Med. Eng. 24 (4), 101-102. doi:10.19338/j.issn.1672-2019.2016.04.045

Zheng, X. M. (2019). Efficacy of trimetazidine combined with Astragalus injection on viral myocarditis. Med. J. Chin. People's Health 31 (9), 11-12. doi:10.3969/j. issn.1672-0369.2019.09.005

Zhu, Q. B., Huang, H. F., Xu, Y., Pan, Z. H., and Xiang, J. H. (2020). Effects of Salviae Miltiorrhizae and Ligustrazine hydrochloride on expression of myocardial enzymes, oxidative stress and inflammatory factors in children with viral myocarditis. Matern. Child Health Care China 35 (2), 259-262. doi:10.19829/j.zgfybj.issn.1001-4411.2020.02.021

Zhuang, Z., Wang, Z. H., Deng, L. H., Zheng, Q., Zheng, G. Q., and Wang, Y. (2019). Astragaloside IV exerts cardioprotection in animal models of viral myocarditis: a preclinical systematic review and meta-analysis. Front. Pharmacol. 10, 1388. doi:10.3389/fphar.2019.01388

Conflict of Interest: The authors declare that the research was conducted in the absence of any commercial or financial relationships that could be construed as a potential conflict of interest.

Copyright () $2021 \mathrm{Wu}$, Deng, Yu, Han, Huang, He, Yan and Wang. This is an openaccess article distributed under the terms of the Creative Commons Attribution License (CC BY). The use, distribution or reproduction in other forums is permitted, provided the original author(s) and the copyright owner(s) are credited and that the original publication in this journal is cited, in accordance with accepted academic practice. No use, distribution or reproduction is permitted which does not comply with these terms. 